\title{
National Academy of Mexico position with regard to the Presidential Decree whereby different regulations of the General Statute of Health are reformed and added, specifically that where the Ministry of Health is conferred the right to design and execute public policies regulating the medicinal and scientific use of Cannabis sativa indica Americana pharmacological derivatives, including tetrahydrocannabinol and its isomers and stereochemical variants, as well as to regulate research and national production thereof
}

\author{
Armando Mansilla-Olivares, Ricardo Plancarte-Sánchez, Fernando Meneses-González, \\ Fermín Valenzuela-Gómez-Gallardo, Joaquín López-Bárcena, Ana Luisa Velasco-Monroy, \\ Gilberto Felipe Vázquez-De Anda and José Ignacio Santos-Preciado \\ Academia Nacional de Medicina de México, Committee of Liaison and Dissemination of Knowledge, Ciudad de México, Mexico
}

\begin{abstract}
As a consequence of the Presidential Decree that reforms and additions the General Statute of Health with regard to medicinal and scientific use of tetrahydrocannabinol, its isomers and stereochemical variants, the National Academy of Medicine of Mexico declares its position in favor of regulating investigation and national production thereof.
\end{abstract}

KEY WORDS: Cannabis. Tetrahydrocannabinol. Cannabis therapeutic use.

Given the importance of the reforms and additions published in the Official Gazette of the Federation on June 19, 2017, regarding the use of tetrahydrocannabinol (THC), as well as that of isomers and stereochemical variants pharmacologically deriving from Cannabis sativa, indica and Americana, the National Academy of Medicine of Mexico, as a federal government advisory body points out that:

I. It is reco gnized, through different studies already published several decades ago, that:

1. Delta-9-tetrahydro-cannabinol (THC) is a psychoactive cannabinoid that modifies perception and is found in the resin produced by the leaves and trunks of the female Cannabis plant.

2. Other cannabinoids, such as cannabidiol, have a pharmacological effect with antipsychotic, analgesic, anticonvulsive, neuroprotective and antiemetic properties; however, articles published on the subject are insufficient and more scientific evidence is required.

3. In humans, there is an endocannabinoid system formed by a group of endogenous receptors (CB1, CB2) located in the central nervous system, in the peripheral nervous system and in tissues associated with the
Correspondence:

Ricardo Plancarte-Sánchez

E-mail: planky2b@yahoo.com.mx
Date of reception: 12-10-2018

Date of acceptance: $23-10-2018$

DOI: 10.24875/GMM.M19000220
Gac Med Mex. 2018;154:632-634

Contents available at PubMed www.gacetamedicademexico.com 
immune system, mainly in the lymph nodes. Therefore, activation of these receptors at the cellular level exerts certain regulatory effect on appetite, pain and behavior of the individual.

4. The enormous development of modern pharmacology has allowed chemical synthesis of cannabinoids, which are presented as an alternative to fight pain and other signs and symptoms that fail to respond or respond partially to traditional pharmacological therapeutics.

5. Cannabinoids are the focus of attention in the media owing to their possible utility in the treatment of refractory epilepsy.

6. Synthetic cannabinoids have been tested in different clinical studies by oral route.

7. There is not enough evidence about Cannabis sativa, indica and Americana, also known as marijuana, having any therapeutic effect when consumed by inhalation (cigarettes and other variants), ingestion (snacks or other variants) or by topical application on the skin.

II. Based on the above, the National Academy of Medicine of Mexico, in the follow-up of reforms and additions published in the Official Gazette of the Federation on June 19, 2017 regarding the use of THC and its pharmacological derivatives, proposes for the use of these substances in epilepsy to be carried out:

1. Based on the development of studies of clinical and pharmacological nature to demonstrate the efficacy of these substances prior to integrating them to daily clinical practice.

2. In patients diagnosed with antiepileptic drugs-refractory epilepsy, under the following considerations:

a) THC should not be considered as firstchoice antiepileptic drug.

b) In case THC is prescribed as an antiepileptic drug, it will be imperative giving patient clinical evolution close follow-up.

c) The prescription of THC in patients with refractory or catastrophic epilepsy can only be done by the neurology specialist, under a protocol-based regimen and patient evolution close clinical monitoring. d) Treatment protocols should be duly registered in the Ministry of Health, an entity that exercises regulation, control and health promotion functions, in order for close follow-up of patients to be carried out.

e) It will correspond to a certified neurologist to decide on the use of THC and its derivatives.

f) It is imperative for the health personnel of public and private institutions to be informed and instructed on the characteristics, mechanisms of action and uses of THC and its derivatives.

g) Patients who are prescribed treatment with $\mathrm{THC}$ should be clinically monitored and their evolution written down in the corresponding medical record at their unit of care.

III. The beneficial therapeutic effects of THC and its derivatives published to date in clinical studies suggest that:

1. The use of THC exerts a low-impact therapeutic effect in the treatment of pain in conditions such as rheumatoid arthritis, fibromyalgia, human immunodeficiency virus infection-associated chronic neuropathic pain, chronic refractory spinal pain, difficult-to-control headaches, spasticity-associated pain in multiple sclerosis and eye pain due to glaucoma.

2. Based on that which is noted in the previous point, its prescription is not recommended for the treatment of pain in this specific group of diseases.

3. Given that published scientific evidence is not conclusive, the development of research protocols on possible therapeutic uses of these substances in other conditions is recommended, in order for solid clinical and scientific evidence to be obtained.

IV. The federal health authority should:

1. Establish a registry of medical specialists authorized to prescribe $\mathrm{THC}$ and its derivatives.

2. Establish a national registry of treatment with $\mathrm{THC}$ and its derivatives, which serves for the control of the use of these substances and as a source of information for clinical and research areas. 
3. In order to improve the control of prescribed drug, establish the health units where THC and its derivatives will be available in order for the medication to be distributed by submitting an application containing an authorized treatment protocol in compliance with the guidelines established in the pharmacovigilance programs.

4. Develop an information campaign directed to public and private institutions health personnel in order to specify prescription and follow-up procedures for THC and its derivatives.
V. The National Academy of Medicine of Mexico recognizes the effort of the federal government and health authorities in the publication of the presidential decree, which will enable for the quality of life of patients with specific diseases to be improved, opening the door for the development of scientific and rigorous research on the therapeutic use of cannabinoids in our country

VI. The National Academy of Medicine of Mexico reiterates its commitment to continue with the discussion on the use of THC and its derivatives, in order to contribute to the Mexican population wellbeing. 